\title{
Interpretation of van der Waals density functionals
}

\author{
Per Hyldgaard, ${ }^{1,2}$ Kristian Berland, ${ }^{1}$ and Elsebeth Schröder ${ }^{1}$ \\ ${ }^{1}$ Department of Microtechnology and Nanoscience, Chalmers University of Technology, SE-412 96 Göteborg, Sweden \\ ${ }^{2}$ Materials Science and Applied Mathematics, Malmö University, Malmö SE-205 06, Sweden \\ (Received 4 July 2014; revised manuscript received 8 August 2014; published 28 August 2014)
}

\begin{abstract}
The nonlocal correlation energy in the van der Waals density functional (vdW-DF) method can be interpreted in terms of a coupling of zero-point energies of characteristic modes of semilocal exchange-correlation (xc) holes. These xc holes reflect the internal functional in the framework of the vdW-DF method. We explore the internal $\mathrm{xc}$ hole components, showing that they share properties with those of the generalized-gradient approximation. We use these results to illustrate the nonlocality in the vdW-DF description and analyze the vdW-DF formulation of nonlocal correlation.
\end{abstract}

DOI: 10.1103/PhysRevB.90.075148

PACS number(s): 31.15.E-, 71.15.Mb

\section{INTRODUCTION}

In a seminal paper [1] Rapcewicz and Ashcroft (RA) highlighted the connections between nonlocal correlations, the exchange-correlation (xc) hole concept [2-7] of density functional theory (DFT), and van der Waals (vdW) forces in the inhomogeneous electron gas. RA introduced a simple physical picture of $\mathrm{vdW}$ binding: electrons and their associated xc holes form neutral pairs in a system resembling condensed, vdW-bounded, atomic matter and experience mutual attraction of a dispersive nature [8-10]. In the RA view, it is the local plasmon that characterizes the interaction components, i.e., electron-xc-hole pairs. The RA picture is supported by a previous study of the nonlinear response in the electron gas [11], predicting strong vdW binding from quantum-fluctuation contributions in the interaction diagram that also underpins an analysis of gradient-corrected correlation [12-16]. In fact, the long-range interaction component is interpreted [17] as reflecting the small-momentum fluctuation components that are extracted to reach a generalized gradient approximation (GGA) in the early formulations [5,6,13,17-21]. Together, Refs. $[1,11,17]$ suggest that one can recover vdW forces in nonlocal functional theories that also incorporate the tremendous progress that GGA represents [22,23].

The Rutgers-Chalmers vdW-DF method [24-40] allows efficient computations of the xc energy in an approximation that seamlessly incorporates nonlocal correlation effects, including vdW forces. The vdW-DF method is gaining recognition for helping to extend the success of nonempirical DFT to sparse matter [41]. The vdW-DF method is free from external parameters and rests only on formal theory input [40], for the local density approximation $[2,42]$ (LDA), and for gradient-corrected exchange $[18,36,43,44]$ in its specification of the plasmon behavior. It also includes a GGA exchange component $[35,38,45-50]$. The choice of vdW-DF exchange can be guided by conservation of the full (nonlocal) xc hole [38] and with such consistent-exchange vdW-DF it is possible to investigate bulk-structure and adsorption problems where interactions are in subtle competition [38,39]. The transferability of vdW-DF has also been probed via comparison with quantum Monte Carlo (QMC) results for hydrogen phases [51] and for water [52]. The vdW-DF method was first tested in non-self-consistent forms [27,29,31,33,53] using GGA calculations of the electron density as input for a post-processing evaluation of the nonlocal correlations. With a formal derivation of forces arising from the nonlocal correlation term [34] and with the introduction of efficient algorithms for computing the vdW-DF energy and forces $[54,55]$, the vdW-DF method today benefits from experience in widespread sparse- and general-matter applications [3941,49,56-67].

The nonempirical vdW-DF-method is built around a semilocal internal (or inner) functional [36,38-40], with xc hole $n_{\mathrm{xc}}^{\mathrm{in}}$, and an evaluation of a nonlocal correlation energy $E_{c}^{\mathrm{nl}}$. The internal functional keeps local exchange and correlation together but limits gradient corrections to exchange. The internal functional was introduced as a concept in Ref. [36] but it underpins all formulations of the general vdW-DF versions $[28,30,31,40]$ since it serves to model the local variation in the plasmon-pole response from which $E_{c}^{\mathrm{nl}}$ is formulated. There have to date only been brief discussions of this internal functional [28,36,39].

The goal of this paper is to present the vdW-DF construction formally both in terms of the internal xc hole and physical pictures. Standard vdW-DF presentations [31,33,34,36,39] start more directly with a plasmon-pole representation of the response. However, there are benefits of tracing the plasmon view back to a discussion of the associated internal functional $\mathrm{xc}$ holes. This makes it possible to discuss the close connection that exists between vdW-DF and the GGA descriptions. Moreover, the emphasis on the internal-functional response allows us to interpret the vdW-DF nonlocal correlation energy in terms of the RA physics picture of vdW forces [1]. In turn, these results allow us to illustrate the mechanisms by which vdW-DF retains a collectivity and nonlocality in its description of the screened response and materials interactions.

The paper is organized as follows. In Sec. II, we present an xc-hole based formulation of the vdW-DF framework. It is meant to give the reader an overview of the vdW-DF method in a self-contained and alternative derivation cast in the concepts that we explore in this paper. In Sec. III, we plot and discuss the internal functional xc-hole components of vdW-DF. Section IV contains a demonstration of the link between the vdW-DF nonlocal correlation energy and the RA physics picture. Finally, Sec. V summarizes the paper, while an appendix details that the vdW-DF rests on a correct longitudinal projection in its description of the electrodynamics coupling. 


\section{THE VDW-DF FRAMEWORK}

To discuss the nature of vdW-DF, we start out by noting that the constraint-based GGA has been enormously successful at describing chemically connected systems, both those that have zero dimensions (such as atoms, molecules, and nanoparticles) and those with one or more macroscopic dimensions (such as wires, surfaces, sheets, and solids) [22,23]. We denote such systems "molecular-type" even if they can be infinitely extended.

Our discussion benefits from considering two or more such molecular-type regions in close vicinity. Figure 1 shows an example with benzene adsorbed on graphene at a separation of $3 \AA$ [37]. The vdW-DF method seeks to extend the GGA success (for an individual fragment) by adding an account of the nonlocal correlations that arise among several such fragments as well as inside the fragments [39,41,49]. Only the coupling mediated by the longitudinal component of the electrodynamical interaction described in the Coulomb gauge, $V \equiv\left|\mathbf{r}_{1}-\mathbf{r}_{2}\right|^{-1}$ is considered. The Coulomb Green function is $G=-4 \pi V$.

The starting point is the exact adiabatic connection formula $[2,5,6](\mathrm{ACF})$ :

$$
\begin{aligned}
E_{\mathrm{xc}}+E_{\mathrm{self}} & =-\int_{0}^{1} d \lambda \int_{0}^{\infty} \frac{d \omega}{2 \pi} \operatorname{Tr}\left[\Im \chi_{\lambda}(\omega) V\right], \\
& =-\int_{0}^{1} d \lambda \int_{0}^{\infty} \frac{d u}{2 \pi} \operatorname{Tr}\left[\chi_{\lambda}(\mathrm{i} u) V\right],
\end{aligned}
$$

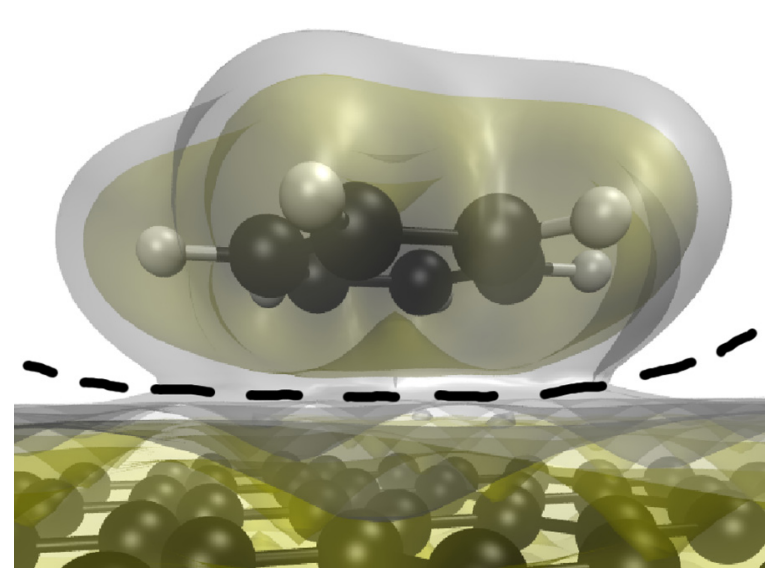

FIG. 1. (Color online) Schematics of a typical problem where vdW-DF is called upon to describe the material binding: a system with multiple molecular-type regions that couple electrodynamically across internal "voids" with sparse electron distribution, Ref. [41]. Each molecular-type fragment can be described by a GGA-type description and such descriptions form the starting point for the vdW-DF evaluation of nonlocal correlations. The voids are not necessarily free of electrons but are regions in which there is only a tail (or overlapping tails) of the molecular-type electron distributions. The schematics is adapted from Fig. 7 of Ref. [37] and shows the atomic configuration and contours of the electron-density distribution of the molecular building blocks for a benzene molecule and a graphene sheet at the vdW-DF binding separation. We note that a natural delineation surface of minimum electron distribution (here illustrated by a dashed curve) runs through interfragment positions with a saddle-point or troughlike behavior in the electron concentration. where $\lambda$ is the electron-electron coupling constant and "Tr" denotes a full trace over the variation [68] in the reducible density-density correlation function $\chi_{\lambda}(i u)$ at imaginary frequency $u$. The infinite self-energy is given by $E_{\text {self }}=$ $(1 / 2) \operatorname{Tr}[V \hat{n}]$, where $\hat{n}(\mathbf{r})$ is the density operator. The reducible density-density correlation function relates an external potential change $\delta \Phi_{\text {ext }}$, at a characteristic frequency $\omega$, to resulting density changes, $\delta n=\chi_{\lambda}(\omega) \delta \Phi_{\text {ext }}$. The electron dynamics causes any such external potential to be screened; the system can also be described by a corresponding screened potential $\delta \Phi_{\text {scr }}$. The irreducible density-density correlation function $\tilde{\chi}_{0}$ relates this screened potential to the same density change, $\delta n=\tilde{\chi}_{\lambda}(\omega) \delta \Phi_{\text {scr. }}$. A Dyson equation relates $\tilde{\chi}_{\lambda}$ and $\chi_{\lambda}$ (see Ref. [6]).

The vdW-DF framework expresses the xc energy

$$
E_{\mathrm{xc}}+E_{\mathrm{self}}=\int_{0}^{\infty} \frac{d u}{2 \pi} \operatorname{Tr}\left[\ln \left(\kappa_{\mathrm{ACF}}(i u)\right)\right],
$$

in terms of an approximation for an effective longitudinal dielectric function $\kappa_{\mathrm{ACF}}(i u)$. We make three observations. First, the expression (2) is formally equivalent to the ACF $[2,5,6]$, as given in Eq. (1), since the coupling-constant integration is captured in the definition of $\kappa_{\mathrm{ACF}}$. Second, the explicit relation is given in terms of an effective externalpotential (density) response function $\chi_{\mathrm{ACF}}(\omega)$ via

$$
-\chi_{\mathrm{ACF}}(\omega) V \equiv 1-\exp \left[-\int_{0}^{1} d \lambda S_{\lambda}(\omega)\right]
$$

where $S_{\lambda}(\omega) \equiv-\chi_{\lambda}(\omega) V$ denotes the fluctuation or plasmon propagator $[69,70]$ at coupling constant $\lambda$. The corresponding longitudinal dielectric function is

$$
\kappa_{\mathrm{ACF}}(i u) \equiv\left[1+\chi_{\mathrm{ACF}}(i u) V\right]^{-1}=\exp \left[\int_{0}^{1} d \lambda S_{\lambda}(\omega)\right]
$$

Third, writing

$$
\kappa_{\mathrm{ACF}}(i u)=\nabla \epsilon(i u) \cdot \nabla G
$$

makes $\kappa$ a rigorously defined longitudinal projection of an effective dielectric function $\epsilon$, as further detailed in Appendix. In vdW-DF, it is assumed that a scalar, but nonlocal, dielectric function, $\epsilon(i u)$ can be used in Eq. (5); there exists a demonstration that such a scalar $\epsilon$ can be constructed for any given xc energy functional (for example, the exact functional) [28,40].

To specify the plasmon-pole description and the xc hole components, we also introduce an effective screened (density) response function $\tilde{\chi}_{\mathrm{ACF}}$ given by

$$
\begin{gathered}
\tilde{\chi}_{\mathrm{ACF}}(i u) V \equiv \kappa_{\mathrm{ACF}}(i u) \chi_{\mathrm{ACF}}(i u) V \\
=\chi_{\mathrm{ACF}}(i u) V \kappa_{\mathrm{ACF}}(i u) \\
=1-\exp \left[\int_{0}^{1} d \lambda S_{\lambda}\right]
\end{gathered}
$$

This definition complies with the Lindhard-type formulation [71]

$$
\kappa_{\mathrm{ACF}}(i u) \equiv 1-\tilde{\chi}_{\mathrm{ACF}} V .
$$


The screened response Eq. (6) is specified via a longitudinal projection

$$
\tilde{\chi}_{\mathrm{ACF}}(i u)=\nabla \alpha(i u) \cdot \nabla
$$

of the local-field dielectric response $\alpha(i u)$. From Eq. (8), it is clear that a scalar approximation for $\alpha$ also specifies the $v d W-D F$ dielectric functional, $\epsilon \equiv 1+4 \pi \alpha$, that enters in Eq. (5) and determines the xc energy Eq. (2) in the vdW-DF framework.

The many-body response nature of any xc functional is naturally expressed in the ACF evaluation of the xc hole

$n_{\mathrm{xc}}\left(\mathbf{r} ; \mathbf{r}^{\prime}\right)=-\frac{2}{n(\mathbf{r})}\left[\int_{0}^{\infty} \frac{d u}{2 \pi} \int_{0}^{1} d \lambda \chi_{\lambda}\left(i u ; \mathbf{r}, \mathbf{r}^{\prime}\right)\right]-\delta\left(\mathbf{r}-\mathbf{r}^{\prime}\right)$,

the electron-deficiency (at $\mathbf{r}^{\prime}$ ) produced around an electron at point $\mathbf{r}$.

For a description of the density functional, it is sufficient to work with the spherically averaged xc hole

$$
\bar{n}_{\mathrm{xc}}\left(\mathbf{r} ; r^{\prime \prime}\right)=\frac{1}{4 \pi\left(r^{\prime \prime}\right)^{2}} \int_{\left|\mathbf{r}^{\prime}-\mathbf{r}\right|=r^{\prime \prime}} d \mathbf{r}^{\prime} n_{\mathrm{xc}}\left(\mathbf{r} ; \mathbf{r}^{\prime}\right) .
$$

The local xc energy per particle $\varepsilon_{\mathrm{xc}}(\mathbf{r})$ is directly related to this $\mathrm{xc}$ hole

$$
\begin{gathered}
E_{\mathrm{xc}} \equiv \int d \mathbf{r} n(\mathbf{r}) \varepsilon_{\mathrm{xc}}(\mathbf{r}) \\
\varepsilon_{\mathrm{xc}}(\mathbf{r}) \equiv \frac{1}{2} \int d \mathbf{r}^{\prime} \frac{n_{\mathrm{xc}}\left(\mathbf{r} ; \mathbf{r}^{\prime}\right)}{\left|\mathbf{r}-\mathbf{r}^{\prime}\right|}=\frac{1}{2} \int_{0}^{\infty} r^{\prime \prime} d r^{\prime \prime} \bar{n}_{\mathrm{xc}}\left(\mathbf{r} ; r^{\prime \prime}\right) .
\end{gathered}
$$

The exact relation

$$
\begin{aligned}
E_{\mathrm{xc}} & =\frac{1}{2} \int d \mathbf{r} n(\mathbf{r}) \int_{0}^{\infty} r^{\prime \prime} d r^{\prime \prime} \bar{n}_{\mathrm{xc}}\left(\mathbf{r} ; r^{\prime \prime}\right) \\
& =\int_{0}^{\infty} \frac{d u}{2 \pi} \operatorname{Tr}\left[\ln \left(\kappa_{\mathrm{ACF}}(i u)\right)\right]-E_{\mathrm{self}}
\end{aligned}
$$

links $\left\langle\mathbf{r}\left|\ln \left(\kappa_{\mathrm{ACF}}\right)\right| \mathbf{r}\right\rangle$ to $\varepsilon_{\mathrm{xc}}(\mathbf{r})$, and hence to an integral over the xc hole.

\section{A. The vdW-DF logic}

A central idea in the vdW-DF framework is to exploit that the formally exact formulation (5) has already made one instance of the electrodynamics coupling $V \propto G$ explicit. One obtains truly nonlocal effects in the xc functional even when using a semilocal GGA-type functional to specify the details of the nonlocal form of $\epsilon$. Accordingly, in the vdW-DF method, we split the total xc energy functional and associated xc holes into semilocal and nonlocal contributions [28],

$$
\begin{gathered}
E_{\mathrm{xc}}[n]=E_{\mathrm{xc}}^{\mathrm{sl}}[n]+\Delta E_{\mathrm{xc}}^{\mathrm{nl}}[n], \\
n_{\mathrm{xc}}\left(\mathbf{r} ; \mathbf{r}^{\prime}-\mathbf{r}\right)=n_{\mathrm{xc}}^{\mathrm{sl}}\left(\mathbf{r} ; \mathbf{r}^{\prime}-\mathbf{r}\right)+\Delta n_{\mathrm{xc}}^{\mathrm{nl}}\left(\mathbf{r} ; \mathbf{r}^{\prime}-\mathbf{r}\right) .
\end{gathered}
$$

The first term $E_{\mathrm{xc}}^{\mathrm{sl}}[n]$ of Eq. (15) is also called the outer semilocal functional and it is given by LDA correlation and a GGA description of gradient-corrected exchange. It would in principle provide an approximate description of a typical GGA problem (i.e., an individual of the molecular-type fragment shown in Fig. 1) because gradient corrections to exchange are typically more important than gradient-corrected correlation $[72,73]$. We shall, for ease of discussion, also refer to such a description as being of a GGA type. At the same time we note that $E_{\mathrm{xc}}^{\text {sl }}$ should not be evaluated in isolation.

The second term $\Delta E_{\mathrm{xc}}^{\mathrm{nl}}$ is viewed as a perturbation [28,40], capturing nonlocal correlation energy from the coupling of plasmon poles that characterize $E_{\mathrm{xc}}^{\mathrm{sl}}$. The formulation of $\Delta E_{\mathrm{xc}}^{\mathrm{nl}} \approx E_{c}^{\mathrm{nl}}$ is, however, in practice based on the use of an internal semilocal functional $E_{\mathrm{xc}}^{\mathrm{in}}[n]$ that is similar to $E_{\mathrm{xc}}^{\mathrm{sl}}[n]$, but with an energy per particle $\varepsilon_{\mathrm{xc}}$ that decreases more rapidly at large values of the scaled density $s=|\nabla n| /\left(6 \pi^{2} n\right)^{1 / 3} / n$. This choice is made to avoid spurious contributions emerging from low-density regions $[1,24,30,38-40]$. The construction via a GGA-type $E_{\mathrm{xc}}^{\text {in }}$ (that also just contains LDA correlation plus GGA gradient-corrected exchange) allows vdW-DF to rest exclusively on formal diagrammatic input $[1,12,17,42]$ while avoiding [34] to explicitly formulate a gradient-corrected correlation term $\delta E_{c}^{\text {grad }}$, which is a necessary but also complex step in the GGA formulations [13,17,19,46,74].

The vdW-DF internal functional $E_{\mathrm{xc}}^{\mathrm{in}}$ is given by a GGAtype internal-functional xc hole $n_{\mathrm{xc}}^{\text {in }} \approx n_{\mathrm{xc}}^{\mathrm{sl}}$ and it is used to introduce an approximate scalar dielectric function $\epsilon$ via

$$
E_{\mathrm{xc}}^{\mathrm{in}}+E_{\mathrm{self}}=\int_{0}^{\infty} \frac{d u}{2 \pi} \operatorname{Tr}\left[\left.\ln (\epsilon(i u))\right|_{\mathrm{grad}}\right] .
$$

This form is motivated by the observation that the longitudinal projection in Eq. (5) becomes redundant in the homogeneous electron gas (HEG) limit. As indicated by the subscript "grad," the $\epsilon$ definition via Eq. (17) rests on an expectation [27,28,33] that this simplification holds approximately true for a weakly perturbed electron gas. Effectively, we write

$$
\begin{aligned}
\epsilon(i u) & =\exp \left[S_{\mathrm{xc}}(i u)\right], \\
E_{\mathrm{xc}}^{\mathrm{in}}+E_{\mathrm{self}} & =\int_{0}^{\infty} \frac{d u}{2 \pi} \operatorname{Tr}\left[S_{\mathrm{xc}}(i u)\right] .
\end{aligned}
$$

The vdW-DF dielectric function (18) is used, via Eq. (9), to also determine an approximation for the full dielectric function $\kappa_{\mathrm{ACF}}$ and hence extend the account to also include nonlocal correlations. Equation (19) is a GGA-guided ansatz for $S_{\mathrm{xc}}$ (and hence $\epsilon$ ) that describes the effective full couplingconstant integration and screening effects within vdW-DF. We note that $S_{\mathrm{xc}}=\ln (\epsilon)$ coincides to linear order with the related approximation $S(\omega) \equiv 1-\epsilon^{-1}(\omega)$ that was used in the vdW-DF method presentation by Dion et al. [31]. References $[28,31,40]$ suggest explicit forms for $S_{\mathrm{xc}}$ (and $S$ ), given in terms of a model plasmon dispersion $\omega_{q}(\mathbf{r})$ at two coordinate points.

Using $S(i u)=1-\exp \left[-S_{\mathrm{xc}}\right] \approx S_{\mathrm{xc}}(i u)$, we interpret the poles $S_{\mathrm{xc}}$ as an approximative specification of the collective modes $\omega_{\eta}$ of the system described by the internal functional Eq. (17), i.e., the zeros of $\operatorname{det}|\epsilon(i u)|$. In the HEG limit, the plasmon-pole dispersion $\omega_{q}$ (entering $S_{\mathrm{xc}}$ ) is the same everywhere and renders a direct specification of $\omega_{\eta}$; in the presence of gradients, the spatial and momentum variation in the $S_{\mathrm{xc}}$ plasmon poles, $\omega_{q}(\mathbf{r})$, represent instead only an approximative specification of the set of internal-functional collective modes $\left\{\omega_{\eta}\right\}$. In any case, these plasmon modes 
are a direct reflection of the shape of the semilocal internalfunctional xc hole, as explained in Sec. II B.

The general-geometry vdW-DF versions [31,36,38] approximate the xc energy

$$
\begin{aligned}
E_{\mathrm{xc}}^{\mathrm{vdW}-\mathrm{DF}}[n] & =E_{\mathrm{xc}}[n]-\delta E_{\mathrm{xc}}[n], \\
& =E_{\mathrm{xc}}^{\mathrm{sl}}+E_{c}^{\mathrm{nl}},
\end{aligned}
$$

where the vdW-DF nonlocal correlation term

$$
\begin{aligned}
E_{c}^{\mathrm{nl}} & \equiv E_{\mathrm{xc}}-E_{\mathrm{xc}}^{\mathrm{in}}=E_{\mathrm{xc}}^{\mathrm{vdW}-\mathrm{DF}}-E_{\mathrm{xc}}^{\mathrm{sl}} \\
& =\int_{0}^{2 \pi} \frac{d u}{2 \pi}\left\{\operatorname{Tr}\left[\ln \left(\kappa_{\mathrm{ACF}}(i u)\right)-\ln (\epsilon(i u))\right]\right\},
\end{aligned}
$$

is evaluated by expanding both terms in the same plasmon-pole description $S_{\mathrm{xc}}$ :

$$
E_{c}^{\mathrm{nl}}=\int_{0}^{\infty} \frac{d u}{4 \pi} \operatorname{Tr}\left[S_{\mathrm{xc}}^{2}-\left(\nabla S_{\mathrm{xc}} \cdot \nabla G\right)^{2}\right] .
$$

This quadratic expansion for $E_{c}^{\mathrm{nl}}$ has the same appearance whether cast in $S_{\text {xc }}$ (as done in Refs. [28,40]) or in terms of $S$ (as done in Refs. [31,41]) because these agree to lowest order.

For given choices of the internal-functional form (and hence of plasmon poles in $S_{\mathrm{xc}}$, Refs. [28,40]) and of $E_{\mathrm{xc}}^{\mathrm{sl}}$ the vdW-DF form generally discards a cross-over term:

$$
\delta E_{\mathrm{xc}}=E_{\mathrm{xc}}^{\mathrm{in}}-E_{\mathrm{xc}}^{\mathrm{sl}} .
$$

An improved alignment between $E_{\mathrm{xc}}^{\mathrm{in}}$ and $E_{\mathrm{xc}}^{\mathrm{sl}}$ minimizes the difference between $E_{\mathrm{xc}}^{\mathrm{vdW}-\mathrm{DF}}$ and an evaluation based on the formal ACF recast Eq. (2). Such an alignment reflects consistency [38] between the plasmon response of the internal functional and that which characterizes $E_{\mathrm{xc}}^{\mathrm{sl}}$, and it is beneficial because it allows the longitudinal projection [in Eqs. (2) and (5)] to leverage an automatic conservation of the full xc hole $[38,39]$.

\section{B. The vdW-DF internal functional specification}

The internal functional is semilocal and of a GGA type. It is specified by LDA exchange energy per particle, $\varepsilon_{x}^{\mathrm{LDA}}(\mathbf{r})=$ $-(3 / 4 \pi) k_{F}(\mathbf{r})$, where $k_{F}(\mathbf{r})=\left(3 \pi^{2} n(\mathbf{r})\right)^{1 / 3}$ denotes the local Fermi wave vector, and an enhancement factor,

$$
\varepsilon_{\mathrm{xc}}^{0}(\mathbf{r})=\varepsilon_{x}^{\mathrm{LDA}}(\mathbf{r}) f_{\mathrm{xc}}^{\mathrm{in}}(n, s)
$$

The internal functional is thus fully given by the local value of the density $n(\mathbf{r})$ and of the scaled density gradients, $s(\mathbf{r})=|\nabla n(\mathbf{r})| / 2 n(\mathbf{r}) k_{F}(\mathbf{r})$. In the vdW-DF design [31,34], the internal functional is exclusively given by the LDA-correlation term $f_{c}^{\mathrm{LDA}}(n)$ (independent of $s$ ) and an exchange gradient enhancement $f_{x}^{\text {in }}(s)$ (independent of $n$ ):

$$
f_{\mathrm{xc}}^{\mathrm{in}}(n, s)=f_{c}^{\mathrm{LDA}}(n)+f_{x}^{\mathrm{in}}(s) .
$$

In the vdW-DF1 [31,32] and vdW-DF-cx [37] versions, we stick with the Langreth-Vosko analysis for screened exchange [18], giving

$$
f_{x}^{\text {in }}=1-\left(\frac{Z_{a b}}{9}\right) s^{2},
$$

specified by $Z_{a b}=-0.8491$. In vdW-DF2 [36], formal scaling analysis $[43,44]$ for pure exchange yields an enhancement of curvature with $Z_{a b}=-1.887$. The form of $f_{\mathrm{xc}}^{\mathrm{LDA}}$ is taken from Ref. [42].

The resulting energy-per-particle expression (25) provides a full specification of a vdW-DF internal xc hole $n_{\mathrm{xc}}^{\text {in }}$ inside a model that assumes a Gaussian spherical average form $[28,40]$

$$
\bar{n}_{\mathrm{xc}}^{\mathrm{in}}(\mathbf{r}, q)=-\exp \left[-\gamma\left(q / q_{0}(\mathbf{r})\right)^{2}\right] .
$$

The simple form enables analytical evaluation for many of the spatial integrations in the resulting description of $E_{c}^{\mathrm{nl}}$. Also, the model form Eq. (28) ensures that $n_{\mathrm{xc}}(\mathbf{r}, q)$ is itself conserved,

$$
\bar{n}_{\mathrm{xc}}^{\mathrm{in}}(\mathbf{r}, q \rightarrow 0)=-1,
$$

for all exchange-enhancement choices in $f_{\mathrm{xc}}^{\text {in }}$ and for any value of $\gamma$. Choosing $\gamma=4 \pi / 9$ in the Gaussian model Eq. (28) provides a simple relation between the inverse length scale [31]

$$
q_{0}[n](\mathbf{r})=k_{\mathrm{F}}[n](\mathbf{r}) f_{\mathrm{xc}}^{\mathrm{in}}[n](\mathbf{r})
$$

of the model hole $n_{\mathrm{xc}}^{\text {in }}$ and the internal functional energy-perparticle variation Eq. (25). This variation in $q_{0}$ is in turn used to formulate the vdW-DF evaluation of $E_{c}^{\mathrm{nl}}$ in terms of a universal kernel $\phi_{\mathrm{nl}}$, as detailed in Refs. [28,31,32,34,40].

An important point for our discussion and interpretation, Sec. IV, is that the shape of the internal semilocal xc hole, given by Eqs. (28) and (30), is used in vdW-DF to determine the local variation in the plasmon poles $\omega_{q}(\mathbf{r})$, Refs. [28,31,40]. The connection is made by noting that the spherical averaged $\mathrm{xc}$ hole $\bar{n}_{\mathrm{xc}}^{\mathrm{in}}(\mathbf{r}, q)$ also defines a natural wave-vector decomposition $[5,6,13,17,18]$ for the internal functional energy per particle,

$$
\begin{gathered}
\varepsilon_{\mathrm{xc}}^{\mathrm{in}}(\mathbf{r})=\int \frac{d \mathbf{q}}{(2 \pi)^{3}} \varepsilon_{\mathrm{xc}}^{\text {in }}(\mathbf{r}, \mathbf{q}), \\
\varepsilon_{\mathrm{xc}}^{\mathrm{in}}(\mathbf{r}, \mathbf{q}) \propto \bar{n}_{\mathrm{xc}}^{\mathrm{in}}(\mathbf{r}, q) / q^{2} .
\end{gathered}
$$

Evaluating the imaginary frequency integral in the formal relation

$$
n(\mathbf{r}) \varepsilon_{\mathrm{xc}}^{\mathrm{in}}(\mathbf{r})=\int_{0}^{\infty} \frac{d u}{2 \pi} S_{\mathrm{xc}}(i u, \mathbf{r}, \mathbf{r})
$$

with the plasmon-pole specification $[28,40]$ for $S_{\mathrm{xc}}(i u)$ yields a wave-vector decomposition [6]

$$
\varepsilon_{\mathrm{xc}}^{\mathrm{in}}(\mathbf{r}, \mathbf{q})=\pi\left(\frac{1}{\omega_{q}(\mathbf{r})}-\frac{2}{q^{2}}\right),
$$

that links $\omega_{q}(\mathbf{r})$ to the chosen description of the internal functional xc hole $n_{\mathrm{xc}}^{\text {in }}(\mathbf{r}, \mathbf{q})$.

\section{INTERNAL-FUNCTIONAL EXCHANGE-CORRELATION HOLES}

In this section, we visualize the vdW-DF internal xc hole and compare it to that of the numerical-GGA constructions $[45,74]$. This casts light on the nature of the vdW-DF since these internal $\mathrm{xc}$ holes define the vdW-DF dielectric function $\epsilon$ from which vdW-DF builds an account of truly nonlocal correlations.

Figure 2 shows the scaled-density gradient $s$ contours of a benzene dimer at binding separation-a typical molecular binding system [38]. The plot documents that a region with low-to-moderate $s$ values exists between the molecular 


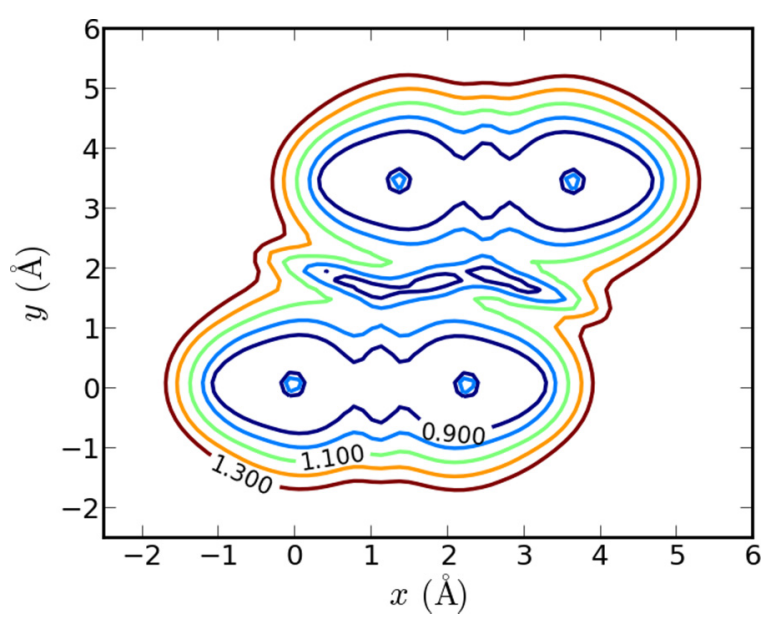

FIG. 2. (Color online) Contours of the scaled density gradients $s=|\nabla n| /\left(2 k_{\mathrm{F}} n\right)$ in a benzene dimer at binding separation. The saddle-point behavior, at low-density and low-to-moderate $s$ values in the region between the molecules, is typical of binding with a significant vdW component, Refs. [37,38].

fragments, where the overlap of two decaying densities causes a saddle-point or troughlike behavior. Nonlocal correlation contributions arise from low-density regions [37] and from low-to-moderate values of the scaled density gradient $s$ [38]. There is no contradiction even if $s$ typically enhances exponentially outside a molecular-type region, because in vdW-DF, the binding from nonlocal correlations arises predominantly in the regions between molecular fragments $[37,62,75]$.

Figure 3 shows the internal functional enhancement factor, Eq. (26) of vdW-DF1 and vdW-DF2. For vdW-DF1, this figure corresponds to density gradient $s<2$ that are often most relevant for $E_{c}^{\mathrm{nl}}$ binding contributions [38].

The spherically averaged real-space $\mathrm{xc}$ hole of the internal functional of vdW-DF is extracted from an inverse Fourier transform of Eq. (28). This real-space internal xc hole is given by the following simple Gaussian form:

$$
\begin{gathered}
\bar{n}_{\mathrm{xc}}^{\mathrm{in}}\left(\mathbf{r} ;\left|\mathbf{r}^{\prime}-\mathbf{r}\right|\right)=-n(\mathbf{r}) J\left(f(\mathbf{r}) ; 2 k_{F}\left|\mathbf{r}-\mathbf{r}^{\prime}\right|\right), \\
J(f, z)=\left(\frac{3}{2}\right)^{4} \frac{f^{3}}{4 \pi} \exp \left[-\frac{(3 f z)^{2}}{64 \pi}\right] .
\end{gathered}
$$

We choose to discuss the role of the internal xc hole in a form scaled with a distance-weighted measure [2]:

$$
\frac{4 \pi z^{2}}{\left(2 k_{F}\right)^{3}} \bar{n}_{\mathrm{xc}}^{\mathrm{in}}(\mathbf{r} ; z)=-\left(\frac{z^{2}}{6 \pi}\right) J(f(\mathbf{r}) ; z)
$$

The weighted expression (37) reflects how the shape of the $\mathrm{xc}$ hole and the electrodynamics coupling $V$ determine the energy-per-particle variation in the corresponding internal semilocal functional, as given by Eq. (13).

Figure 4 shows a contour plot of the internal $\mathrm{xc}$ hole $\bar{n}_{\mathrm{xc}}(n(\mathbf{r}), s(\mathbf{r}))$ as defined by Eq. (26) and weighted and scaled according to Eq. (37). The plot represents the behavior of both vdW-DF1/vdW-DF-cx and vdW-DF2, since the vertical axis is simply the value of the internal functional enhancement factor, $f_{\mathrm{xc}}^{\text {in }}$. The horizontal axis represents the scaled distance $z=2 k_{F}\left|\mathbf{r}^{\prime}-\mathbf{r}\right|$ from the electron position (hole center).
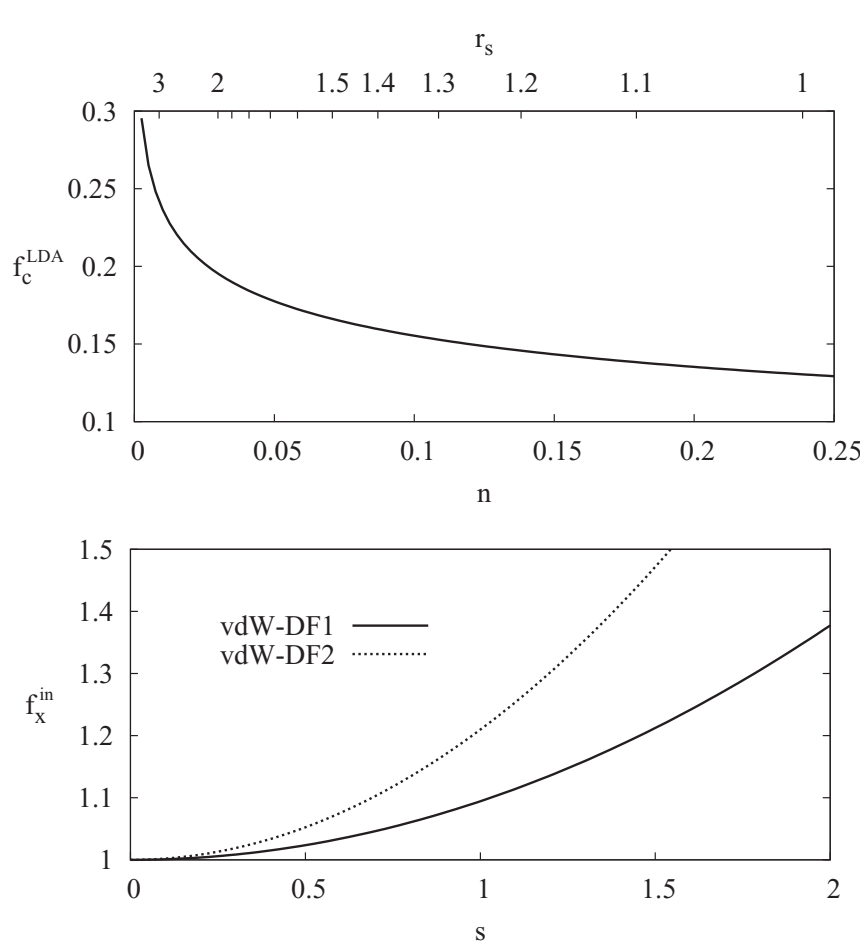

FIG. 3. Correlation (top) and exchange (bottom ) related components of the vdW-DF1 and vdW-DF2 internal functional enhancement factor $f_{\mathrm{xc}}^{\text {in }}=f_{c}^{\mathrm{LDA}}+f_{x}^{\mathrm{in}}$. The first is a function of the density $n$ [or, equivalently, of $\left.r_{s}=(3 / 4 \pi / n)^{1 / 3}\right]$. The second is a function of the scaled density gradient $s=|\nabla n| /\left(2 k_{\mathrm{F}} n\right)$.

Figure 5 compares the internal functional xc hole $n_{\mathrm{xc}}^{\text {in }}$ against the Perdew-Wang (PW) xc hole model for the homogeneous electron gas [76]. This model captures the salient nonoscillatory features of the correlation hole and therefore

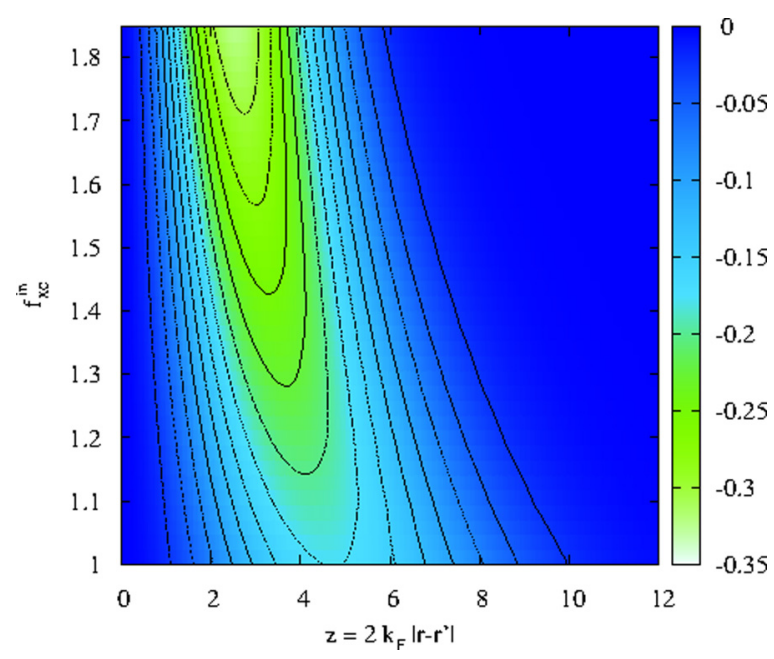

FIG. 4. (Color online) Contour plot of the vdW-DF internal functional xc hole $\bar{n}_{\mathrm{xc}}^{\mathrm{in}}$, spatially weighted and scaled according to Eq. (37). The hole is mapped as a function of scaled separation $z=$ $2 k_{F}\left|\mathbf{r}^{\prime}-\mathbf{r}\right|$ and the characteristic internal functional enhancement factor $f(\mathbf{r})=f_{\mathrm{xc}}^{0}(\mathbf{r})=q_{0}[n](\mathbf{r}) / k_{F}[n](\mathbf{r})$, which is fixed for a given density $n(\mathbf{r})$ and a given scaled density gradient $s=|\nabla n| /\left(2 k_{\mathrm{F}} n\right)$. Contour spacing is 0.025 . 


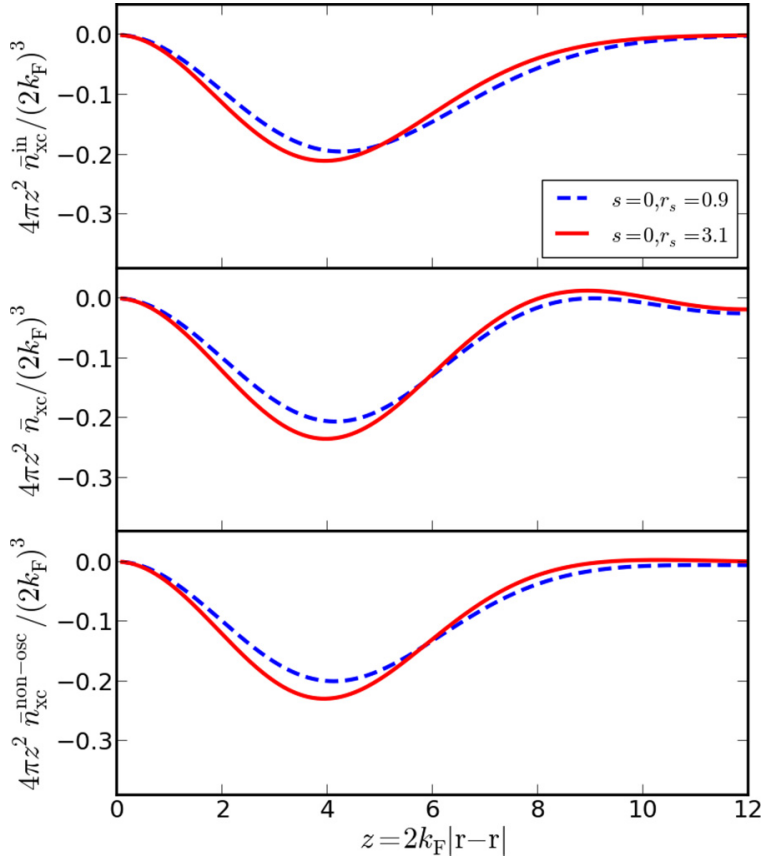

FIG. 5. (Color online) Comparison in the homogeneous limit between the vdW-DF representation of the internal xc hole (top panel) and the Perdew and Wang [76] xc models (mid and bottom panels). Two electron densities, as specified by value of $r_{s}=(3 / 4 \pi / n)^{1 / 3}$ are considered. The middle panel relies on the exact exchange hole while the lower panel relies on a nonoscillatory approximation.

compares well with QMC calculations. In the bottom panel, the nonoscillatory approximation for the exchange hole is used [76]. The holes are plotted as functions of the scaled distances and weighted by the radial measure as earlier. The comparison is shown for two values of the Wigner-Seitz radius $r_{s}=$ $(3 / 4 \pi / n)^{1 / 3}$. The value of $r_{s}=0.9$ corresponds to the density between two neighboring $\mathrm{C}$ atoms of a benzene molecule, while the value of $r_{s}=3.1$ corresponds to the density $1.5 \AA$ out of the benzene-plane above these two $\mathrm{C}$ atoms.

The PW model agrees well with the internal functional xc hole. In particular, the agreement is strikingly similar to the xc hole relying a nonoscillatory approximation for the exchange hole, with the exception of a slightly different trend with changing $r_{s}$. In summary, vdW-DF not only keeps a good balance between local exchange and correlation contributions, in line with the DFT tradition [6], but also an internal xc hole form in fair agreement with QMC.

Equally interesting is the question if the vdW-DF internalhole characterization also remains useful when applied to typical systems. These have density gradients and we need to selectively add the effects of gradient-corrected exchange in $n_{\mathrm{xc}}^{\text {in }}$ (by the vdW-DF design logic). The shape of the vdW-DF model internal xc hole should remain reasonable at values of the scaled gradient $s$ that are deemed relevant for the evaluation of the nonlocal correlation.

Numerical GGA $[45,73,74]$ is a well defined procedure to impose charge conservation and a negativity condition on the xc hole of a gradient expansion [12-14,16,17,19,20] around a homogeneous electron gas. Since this xc hole construction can be used to derive popular xc functionals such

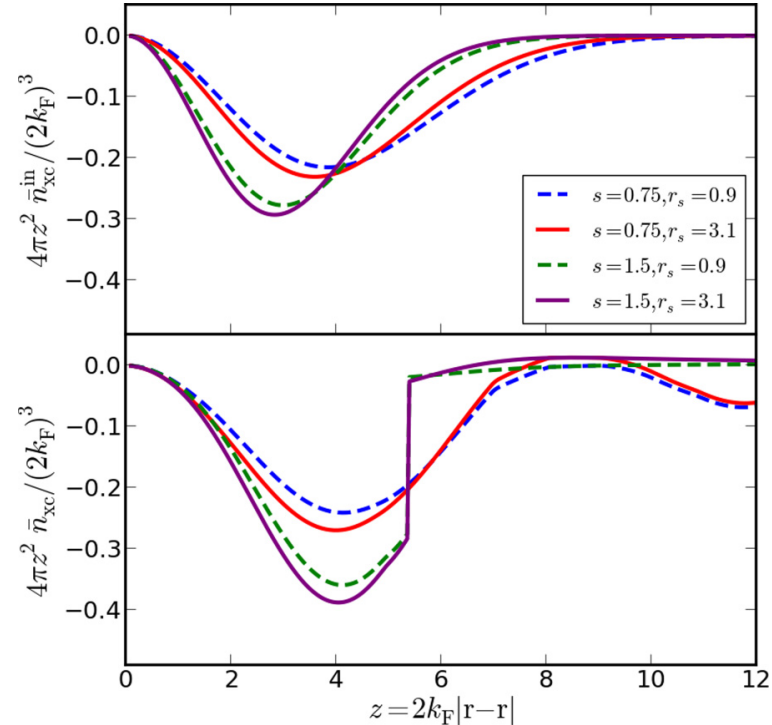

FIG. 6. (Color online) A comparison of the vdW-DF2 internal xc hole and a numerical xc hole consisting of exchange at the GGA level and correlation at the LDA level. The scaled gradient is chosen as $s=0.75$ and 1.5 for two different densities. The hole deepens and narrows as $s$ increases.

as PW86, PBE, and PBEsol [45,46,77], we compare relevant components (including gradient corrected exchange but not gradient-corrected correlations) of also these to those of the $\mathrm{vdW}-\mathrm{DF}$ internal functional at relevant nonzero values of the density gradient.

Figure 6 compares the vdW-DF2 internal functional xchole representations at $s=0.75$ and 1.5 with the numericalGGA specification of Ref. [74]. For the correlation, only the LDA part of the numerical xc hole is included, since only this component is used in the construction of the internal xc-hole of vdW-DF. The vdW-DF2 version is chosen for comparison, because its internal functional has the same small-s behavior as the PBE functional [46] and the numerical hole construction of Ref. [74] leads to a GGA xc that resembles PBE. Conservation is built into the vdW-DF model of the internal xc hole (for any choice of $f_{\mathrm{xc}}^{\text {in }}$, Eq. (29) and there is no need to enforce hard cutoffs as in the numerical GGA construction [40].

Comparing the two panels of Fig. 6 with those of Fig. 5, we see that in all cases the holes become deeper and shorter-ranged as $s$ increases. However, whereas the two holes agree fairly well for small $s$, their shapes grow dissimilar as $s$ increases. The agreement with the numerical-GGA construction is best when (as is more relevant for larger and flatter fragments close to binding separation [38]) we can limit the value of the scaled gradient to $s<1$.

\section{INTERPRETATION OF THE VDW-DF NONLOCAL CORRELATION ENERGY}

In this section, we show that the vdW-DF method relies on a nonlocal-correlation formulation that can be interpreted as an implementation of the RA picture of vdW forces [1]. As mentioned in the introduction, this picture sees nonlocal correlations as arising from an electrodynamical coupling of, 
originally independent, $\mathrm{xc}$ holes of a traditional semilocal functional description; in vdW-DF represented by the GGAtype internal xc functional holes $n_{\mathrm{xc}}^{\text {in }}$. The vdW-DF nonlocal correlation term $E_{c}^{\mathrm{nl}}$ represents a counting of coupling-induced zero-point energy shifts of the characteristic plasmon modes of these internal-functional xc holes $[1,24,40]$. We provide the interpretation by adapting the analysis that Mahan used to discuss the nature of $\mathrm{vdW}$ forces and detail their relation to Casimir forces [10,78].

To begin, we formulate the exact xc energy (2)

$$
\begin{aligned}
E_{\mathrm{xc}}[n]+E_{\mathrm{self}}[n] & =\int_{-\infty}^{\infty} \frac{d u}{4 \pi} \ln (\Delta(y=i u)), \\
\Delta(y) & \equiv \operatorname{det}\left|\kappa_{\mathrm{ACF}}(y)\right|,
\end{aligned}
$$

where time-reversal symmetry has been used to extend the integration over $u$ to $-\infty$. The right-hand side of Eq. (38) can be evaluated from a contour around the complex-frequency plane that runs down the imaginary axis and closes around the half-plane of positive frequencies. This contour picks up characteristic poles of det $\left|\kappa_{\mathrm{ACF}}\right|$ with simple residues, as is evident in the rewrite [10]

$$
\frac{1}{4 i \pi} \oint_{c} \frac{z d z}{\Delta(z)} \frac{\partial}{\partial z} \Delta(z) .
$$

The form (40) counts the sum of collective modes, given by $\Delta\left(y_{\eta}\right)=0$. With a general specification of $\kappa_{\mathrm{ACF}}(i u)=1-$ $\tilde{\chi}_{\mathrm{ACF}}(i u) V$ (beyond the approximation used in the vdW-DF versions) there will also be corrections from poles in $\partial \Delta(z) / \partial z$. This second set of poles corresponds to singularities in the local-field response [10] $\tilde{\chi}_{\mathrm{ACF}}$. Such singularities are normally associated with particle-hole excitations [10,14,79].

The nonlocal correlation term (22) is formulated as a difference between the xc energy when defined in terms of $\ln \kappa_{\mathrm{ACF}}$ and $\ln \epsilon$. It therefore expresses an xc energy shift produced by an electrodynamical coupling. The general result (38) allows us to discuss the nature of this coupling-induced energy shift.

We first consider a single molecular-type fragment, i.e., one of the molecular-type regions in Fig. 1. We use $\omega_{\eta}$ and $\bar{\omega}_{\eta}$ to denote the collective modes of the internal and of the full $\mathrm{vdW}-\mathrm{DF} \mathrm{xc}$ functional, given respectively by $\operatorname{det}\left|\epsilon\left(\omega_{\eta}\right)\right|=0$ and det $\left|\kappa_{\mathrm{ACF}}\left(\bar{\omega}_{\eta}\right)\right|=0$. The contour evaluation (40) provides the formal evaluation,

$$
E_{c}^{\mathrm{nl}}=\frac{1}{2} \sum_{\eta}\left(\bar{\omega}_{\eta}-\omega_{\eta}\right),
$$

since, as motivated below, we can ignore contributions from particle-hole excitations [6].

The vdW-DF idea of expressing all functional components through an analysis of the response in an internal (GGA-type) functional is what makes Eq. (41) relevant for analyzing $E_{c}^{\mathrm{nl}}$. A key observation is that the specification of the vdW-DF internal functional keeps local exchange and local correlation together to allow a cancellation of terms arising from particle-hole excitations [6]. Accordingly, Ref. [31] uses simply a plasmonpole representation for $S(i u) \equiv 1-\epsilon(i u)^{-1}$ but leaves no room for singularities directly in $\epsilon$. The same observation underpins our assumption, in Sec. II, that all singularities in the internal-functional specification $[28,40] S_{\mathrm{xc}}(i u)=\ln [\epsilon(i u)]$ should be seen as exclusively reflecting collective (plasmon) poles of $\epsilon(i u)$. Moreover, while Eq. (4) ensures that the reducible response function $\chi_{\mathrm{ACF}}$ has singularities at the collective modes of $\kappa_{\mathrm{ACF}}(i u)$, there can be no single-particle singularities in the irreducible response $\tilde{\chi}_{\mathrm{ACF}}$. This follows in the vdW-DF framework (and only there) because $\tilde{\chi}_{\mathrm{ACF}}$ is set by the internal functional behavior, through $\epsilon=1+4 \pi \alpha$ and Eqs. (18) and (9).

The formal evaluation in Eq. (41) can be used to interpret the vdW-DF nonlocal correlation term, Eq. (22), as an implementation of the RA picture of $\mathrm{vdW}$ forces [1]. The $E_{c}^{\mathrm{nl}}$ term tracks changes in characteristic plasmon modes of the system as described in vdW-DF and in a semilocal functional defined by xc functional holes $n_{\mathrm{xc}}^{\text {in }}$ (with a partial GGA character). Inclusion of the electrodynamical coupling changes the dielectric functions and hence the characteristic plasmon modes that characterize $n_{\mathrm{xc}}^{\text {in }}$. In effect, the energy shift in Eq. (41) tracks the effects of coupling the GGA-type internal functional holes.

It is also interesting to compare the formal framework of the vdW-DF method and the random phase approximation (RPA) [6,79-83]. The RPA correlation energy is [6]

$$
E_{c}^{\mathrm{RPA}}=\int_{0}^{\infty} \frac{d u}{2 \pi} \operatorname{Tr}\left\{\ln \left(1-\tilde{\chi}_{0} V\right)\right\}+\tilde{\chi}_{0} V .
$$

Our comparison will be based on the full $E_{c}^{\mathrm{nl}}$ representation (22) that underpins Eq. (41) and was used in an early seamless functional for layered structures [29]. We shall in Sec. $\mathrm{V}$ return to a discussion of RPA and vdW-DF, keeping in mind also that the recent vdW-DF versions $[31,36,38]$ use a second-order expansion of Eq. (22) in $S_{\mathrm{xc}}$. There are formal similarities between RPA and the vdW-DF method. The vdW-DF framework, Eqs. (2) and (5), builds on the exact ACF as does RPA; in fact, the RPA xc energy is obtained by inserting the approximation $\kappa_{\mathrm{RPA}}=1-\tilde{\chi}_{0} V$ in Eq. (2). Also, the RPA correlation energy can be exactly reformulated [79,81],

$$
E_{c}^{\mathrm{RPA}}=\frac{1}{2} \sum_{n}\left(\Omega_{n 0}-\Omega_{n 0}^{D}\right),
$$

where $\Omega_{n 0}^{D}\left(\Omega_{n 0}\right)$ denotes a RPA excitation energy as described to lowest (full) order in $\lambda$. The RPA interpretation as a counting of zero-point energy shifts, Eq. (43), resembles the interpretation (41) that we present for the nonlocal-correlation energy in the vdW-DF method. There are also fundamental differences. The RPA crafts $\tilde{\chi}_{0}$ from particle-hole excitations, typically given by Kohn-Sham orbitals and energies [81-83], whereas vdW-DF proceeds by asserting its response description through a plasmon model $[29,31,34]$. The summation in the vdW-DF $E_{c}^{\mathrm{nl}}$ interpretation (41) is restricted to zero-point energy contributions defined by collective modes.

Additional details about the nature of the vdW-DF nonlocal correlation term $E_{c}^{\mathrm{nl}}$ can be obtained by considering the case of two molecular fragments, $A$ and $B$, separated by a delineation surface as illustrated in Fig. 1. In the following, we focus exclusively on the binding that arises in the nonlocalcorrelation component of $E_{\mathrm{xc}}$, noting that there will also be other interaction components (arising through the interplay 
between kinetic-energy repulsion, Coulomb terms, and in the outer semilocal functional $E_{\mathrm{xc}}^{0}$ ). The analysis applies also when, as in weak chemisorption, there is some density overlap, as we can proceed within a superposition-of-density scheme $[53,84]$. The analysis is not relevant for cases where there are also chemical bonds across the delineation surface but we defer a discussion of limitations until we can formulate this in terms of criteria on the electron-response description.

To make the discussion more specific, we cast the interpretation in terms of explicit approximations. We let $n_{A}$ (and $n_{B}$ ) denote the density of fragment $A(B)$ when treated in isolation. These densities should be seen as DFT solutions as obtained in a vdW-DF version; note that $n_{A}$ extends into the area that the delineation surfaces assign as region $B$ and vice versa. We assume that the multicomponent density can be sufficiently approximated as a sum of fragment densities, $n=n_{A}+n_{B}$. This is a general approximation scheme[84] which is often accurate for systems held together by dispersive forces in competition with other interactions [53]. From separate densities $n_{A}$ and $n_{B}$ we can define per-fragment screened response functions $\tilde{\chi}_{\mathrm{ACF}}^{*, A}$ and $\tilde{\chi}_{\mathrm{ACF}}^{*, B}$. We note that the corresponding reducible response function $\chi_{\mathrm{ACF}}^{*, A}$ must have singularities at the vdW-DF collective modes $\bar{\omega}_{A}$ for fragment $A$. The same goes for the description of fragment $B$. Next, we introduce $\tilde{\chi}_{\mathrm{ACF}}^{A}$ as the region-projected part of this irreducible response, i.e., the matrix formed from $\tilde{\chi}_{\mathrm{ACF}}^{*, A}$ by restricting both coordinates to reside in delineated region " $A$ " as well as corresponding projections for the reducible response function $\chi_{\mathrm{ACF}}^{A}$ and for $\kappa_{\mathrm{ACF}}^{A}$.

At this stage we can discuss the limitations on the extended $E_{c}^{\mathrm{nl}}$ analysis presented below. One requirement is that we approximately retain a Dyson-like link, as in Eq. (6), among the response descriptions even when working with the fragmentprojected response description:

$$
\chi_{\mathrm{ACF}}^{A(B)} \approx\left[\kappa_{\mathrm{ACF}}^{A(B)}\right]^{-1} \tilde{\chi}_{\mathrm{ACF}}^{A(B)} .
$$

A second, related, requirement is that the collective modes $\bar{\omega}_{A(B)}$ also represent the poles of $\chi_{\mathrm{ACF}}^{A(B)}$. This second requirement can be formulated as the condition that det $\left|\kappa_{\mathrm{ACF}}^{*, A(B)}(i u)\right|$ (where the determinant reflects an integration over the entire space) has the same zeros as is found for det $\left|\kappa_{\mathrm{ACF}}^{A(B)}(i u)\right|_{A(B)}$ (where the determinant range is limited to the delineated region). The conditions can only hold approximately except when discussing well-separated fragments.

Notwithstanding the requirements for using a partitioning scheme, we proceed to deepen our analysis of the nonlocal correlation term. Such a scheme has also been used, for example, to extract an asymptotically exact evaluation of interactions among defects on a surface supporting a metallic surface state [85-91]. The important part of the Coulomb coupling is in this problem the component $V_{A B}$ of the Coulomb term that connects a point in the delineated region $A$ with a point in the other region $B$. Using a simple matrix factorization of det $\left|\kappa_{\mathrm{ACF}}\right|$ (and of det $|\epsilon|$ ), we thus obtain

$$
\begin{gathered}
E_{\mathrm{xc}}^{\mathrm{nl}, \mathrm{AB}} \approx \int_{-\infty}^{\infty} \frac{d u}{4 \pi} \ln \left(\Delta^{*}(i u)\right), \\
\Delta^{*}(i u) \equiv \operatorname{det}\left|1-\chi_{\mathrm{ACF}}^{A}(i u) V_{A B} \chi_{\mathrm{ACF}}^{B}(i u) V_{B A}\right| .
\end{gathered}
$$

We note in passing that the result of Eq. (45) is consistent with the traditional result for the asymptotic vdW binding $[24,25]$

$$
E_{\mathrm{vdW}}(d) \equiv-\int_{0}^{\infty} \frac{d u}{2 \pi} \operatorname{Tr}\left[\boldsymbol{\alpha}_{\mathrm{ext}}^{A}(i u) T_{A B} \boldsymbol{\alpha}_{\mathrm{ext}}^{B}(i u) T_{B A}\right],
$$

where $T_{A B}=-\nabla_{\mathbf{r}_{a}} \nabla_{\mathbf{r}_{b}}\left|\mathbf{r}_{a}-\mathbf{r}_{b}\right|$ denotes a dipole-dipole coupling tensor between points in separate regions and where $\boldsymbol{\alpha}_{\text {ext }}^{A(B)}$ denotes the external-field susceptibility of fragment $A$ (or fragment $B$ ). The connection between Eq. (45) and Eq. (47) is made by expanding the logarithm and noting that

$$
\chi_{\mathrm{ACF}}^{A(B)}(\omega)=\nabla \cdot \boldsymbol{\alpha}_{\mathrm{ext}}^{A(B)}(\omega) \cdot \nabla
$$

specifies the vdW-DF approximation for these susceptibilities [28].

For a discussion of the electrodynamical coupling expressed in $E_{c}^{\mathrm{nl}}$ we provide a contour-integral evaluation of Eq. (45). Using the contour-integration formulation (40) for $\Delta^{*}$, we now have contributions from the poles $\bar{\omega}_{\eta_{A(B)}}$ of the fragment response function $\chi_{\mathrm{ACF}}^{A(B)}$. We assume that these give rise to contributions that resemble those specified by the exciton-susceptibility tensors in Ref. [10]. Equally important, the form Eq. (45) has regular plasmon-pole contributions given by the zeros $\bar{\omega}_{\left(\eta_{a}, \eta_{B}\right)}$ of

$$
\Delta^{*}(\omega)=\operatorname{det}\left|1-\boldsymbol{\alpha}_{\text {ext }}^{A}(\omega) T_{A B} \boldsymbol{\alpha}_{\text {ext }}^{B}(\omega) T_{B A}\right| .
$$

Adapting the argument presented in Ref. [10], these interfragment collective mode $\bar{\omega}_{\left(\eta_{a}, \eta_{B}\right)}$ correspond to a coupling between polarizability contributions defined in $\boldsymbol{\alpha}_{\text {ext }}^{A}(\omega)$ and $\boldsymbol{\alpha}_{\text {ext }}^{B}(\omega)$ by modes $\bar{\omega}_{\eta_{a}}$ and $\bar{\omega}_{\eta_{B}}$. Overall the coupling contour integration leads to an approximative evaluation

$$
E_{\mathrm{xc}}^{\mathrm{nl}, A B} \approx \frac{1}{2} \sum_{\eta_{A}, \eta_{B}}\left(\bar{\omega}_{\left(\eta_{A}, \eta_{B}\right)}-\bar{\omega}_{\eta_{A}}-\bar{\omega}_{\eta_{B}}\right),
$$

and establishes a further link between the nonlocal-correlation term in vdW-DF and the RA picture, viewing vdW forces as arising as a coupling of (semilocal, initially independent) $\mathrm{xc}$ holes $[1,11,24]$.

Finally, we note that we can extend a partition-based analysis also to the case when there are three (or more) molecular fragments, again adopting the analysis used for the study of electronic substrate-mediate interactions among defects on surfaces [86,89]. For cases with three moleculartype fragments, denoted $A, B$, and $C$, we find

$$
\begin{aligned}
& E_{\mathrm{xc}}^{\mathrm{vdW}-\mathrm{DF}}+E_{\mathrm{self}} \\
& \approx-\sum_{i=A, B, C} \int_{0}^{\infty} \frac{d u}{2 \pi} \operatorname{Tr}\left[\ln \left(1+\chi_{\mathrm{ACF}}^{i} V_{i i}\right)\right] \\
& +\int_{0}^{\infty} \frac{d u}{2 \pi} \operatorname{Tr}\left[\operatorname { l n } \left(1-\sum_{i<j}^{A, B, C} \chi_{\mathrm{ACF}}^{i} V_{i j} \chi_{\mathrm{ACF}}^{j} V_{j i}\right.\right. \\
& \left.\left.\quad-2 \chi_{\mathrm{ACF}}^{A} V_{A B} \chi_{\mathrm{ACF}}^{B} V_{B C} \chi_{\mathrm{ACF}}^{C} V_{C A}\right)\right]
\end{aligned}
$$

The trio term does not naturally enter in the vdW-DF description when investigating a system with only two 
molecular-type regions near binding separation. The argument for approximating $\chi_{\mathrm{ACF}}^{i}$ as exclusively connecting two points inside the same fragment breaks down if one were to partition an individual molecular-type region.

\section{DISCUSSION}

The influence of screening and nonadditivity effects on the $\mathrm{vdW}$ forces are explored in a significant body of literature, for example in Refs. [1,92-106]. In this section, we discuss to what extent the vdW-DF method $[31,36,38]$ can capture such effects. In particular, we first compare vdW-DF to the RPA for the correlation energy and then discuss vdW-DF in light of a recently suggested classification scheme of dispersion interaction effects that lies beyond pair-wise summations [106].

The vdW-DF method shares with RPA an electron-based foundation as they avoid partitioning into, for example, atomic components. The methods also share a zero-point-energy counting nature (Sec. IV), an emphasis on approximating the ACF through longitudinal dielectrical functions that comply with the continuity equation (see Appendix), and conservation of the associated xc hole [31,38-40]. One difference is that the vdW-DF is based on a fully screened response description via a plasmon-based starting point that reflects a GGA-type internal-functional xc hole, whereas in RPA one starts with independent-particle excitations [81-83]. With the full $E_{c}^{\mathrm{nl}}$ expression (22), used in an early seamless vdW-DF functiona [27,29], the vdW-DF method relies on the same machinery as RPA for systematically including screening effects, namely the Dyson equation for the density-density correlation function [6], as shown in Sec. II.

At the same time, the popular general-geometry vdW-DF versions and closely related variants $[31,35,36,38,49,50]$ rest on a second-order expansion (23) of $E_{c}^{\text {nl }}$, a step that is not used in RPA calculations. An interesting question is then how much of the screening, collectivity, and nonadditivity effects are retained after making this truncation. The question is complex and we limit the discussion to making some comments in the context of a recent perspective article by Dobson [106].

Dobson classifies nonadditivity effects as follows. Class A contains the effects of bond formation. These effects are automatically included in vdW-DF. Class B is the spectator effect, that is, the modification by an additional moleculartype fragment on the electrodynamics coupling between two molecular-type fragments. Class $\mathrm{C}$ contains many-body effects that result with nondegenerate electron states and their ability to enhance the electronic response. A consequence is, for example, different asymptotic scaling laws for the $\mathrm{vdW}$ attraction between sheets of metals or among metallic nanotubes than between insulators [93-97,99,103,105,106]. Classes B and C are expected to be of greater importance for asymptotic interactions than at binding separations where there are contributions from many plasmons $[37,99,105]$.

For a vdW-DF version to fully address nonadditivity effects of class $B$, it requires that the evaluation proceeds with the full interaction form [27,29], not the expansion (23) used for the more recent and popular vdW-DF versions [31,36,38]. However, vdW-DF reflects multipole enhancements in the binding among molecules [57,59] and image-plane formation in the binding of carbon nanotubes [107] and in challenging physisorption problems [108,109]. Image-plane effects are captured in those recent expanded vdW-DF versions through the stronger sensitivity to the low-density regions arising at surfaces than to the high-density regions of the bulk [37].

To fully capture effects in class $\mathrm{C}$ one would also need to refine the vdW-DF inner-functional response model beyond a simple plasmon model relying on a GGA-based account. However, some of the energetic impact of these effects is also, in practice and at a cruder level, reflected in the modern vdWDF versions. In low-density, highly homogeneous systems, typical of a metal surface [108,109], the vdW-DF plasmon model yields small excitation energies, strongly enhancing nonlocal correlation effects. On the other hand, except at edge regions, the GGA-based construction of vdW-DF does not distinguish between a limited molecular-type fragment, such as the center part of a polyaromatic hydrocarbon, and an extended fragment that has no gap, such as graphene or a metallic nanotube. This is a distinction that becomes important at asymptotic separations between fragments [97,99,103].

The recent vdW-DF versions [31,36,38] are formulated with the expectation that the second-order expansion (23) is often sufficient in binding situations, with two moleculartype fragments in close proximity [110]. At binding it is important to treat truly nonlocal correlation effects and the more local/semilocal correlation effects on a same footing $[28,31,34,37]$. In the sections above, we have illustrated that vdW-DF should not be viewed merely as a summation of contributions from pairs of density points. It is rather an expression of coupling of semilocal xc holes with a finite extension and with a shape and dynamics that already reflect a GGA-type response behavior. The internal functional xc holes express a collectivity that generally extends beyond that of a single atom and represents a GGA-level of screening that we assume is often adequate for treating interfragment binding.

The good performance of vdW-DF, in particular for the most recent nonempirical versions and variants, indicates that the vdW-DF method is capable of accurately reflecting the complicated balance that can exist between general interaction contributions [37,39,108,109]. For example, the recent consistent-exchange vdW-DF-cx version [38] can correctly describe the competition between covalent and ionic bonds in ferroelectrics, and between exchange effects and ionic and vdW attraction in weak-organic chemisorption [39]. One needs Axilrod-Teller [1,92] corrections (51) and beyond $[27,29,81,100]$ to fully characterize the general dispersive interaction in systems that have three or more molecular-type regions. However, when two molecular-type regions are at their binding separation there is not generally room for a third molecule to get close and significantly influence that coupling.

Finally, for a quantitative discussion of what screening effects are retained in recent vdW-DF versions one needs to compare the results of these versions with those obtained when using the full $E_{c}^{\mathrm{nl}}$ form [27,29] under the same approximation for the internal-functional description. Two of us have led one early such exploration [111] but no conclusion can be reached in that study because we refined the plasmon model between the layered-geometry formulation of Ref. [29] and the launching of vdW-DF1 [31]. A comparison of the results based on the modern $S_{\mathrm{xc}}$ response form is beyond the present scope. 


\section{SUMMARY}

Several formal properties of the vdW-DF theory have been highlighted. Specifically, we have documented how an effective internal xc hole can be viewed as a central building block in obtaining the nonlocal correlation of vdW-DF. We have documented how this internal $\mathrm{xc}$ hole resembles the $\mathrm{xc}$ hole construction that underpins standard GGA descriptions. Further, we have argued how the nonlocal correlations in $\mathrm{vdW}-\mathrm{DF}$ can be interpreted as arising from the shift in collective modes induced by the electrodynamical coupling between such xc holes. This argument connects vdW-DF to the well-established RA picture of vdW-DF interactions. Finally, we have compared the vdW-DF method to RPA. By discussing the formal properties of vdW-DF and links to other theories, we hope to help build bridges that stimulate the dissemination of ideas, not just within the field of van der Waals interactions but also within the wider field of material modeling.

\section{ACKNOWLEDGMENTS}

The authors are grateful for many insightful discussions with David C. Langreth, who passed away in 2011, and Bengt I. Lundqvist. The authors are furthermore grateful for input, insight, and encouragement from G. D. Mahan, primarily during his two extended visits to Chalmers, in 2009 and 2011. This work was supported by the Swedish research council (VR) and the Chalmers Area of Advance Materials theory initiative.

\section{APPENDIX: ROLE OF THE CONTINUITY EQUATION IN VDW-DF}

This appendix details the correct longitudinal projection in electrodynamics and is based on notes by and discussions with D.C. Langreth. It serves to further motivate writing the xc energy

$$
E_{\mathrm{xc}}+E_{\mathrm{self}}=\int_{0}^{\infty} \frac{d u}{2 \pi} \operatorname{Tr}[\ln (\nabla \epsilon(i u) \cdot \nabla G)]
$$

so that it expresses the exact longitudinal projection. The form (A1) reflects the continuity equation as well as the constituent equations of the electrodynamical response in materials.

Consider Ohm's law for the current $\mathbf{j}^{\text {ind }}$ induced by a local field $\mathbf{E}=-\nabla \Phi_{\text {loc }}$,

$$
\mathbf{j}^{\text {ind }}(\mathbf{r}, \omega)=\int d \mathbf{r}^{\prime} \boldsymbol{\sigma}\left(\mathbf{r}, \mathbf{r}^{\prime}, \omega\right) \mathbf{E}\left(\mathbf{r}^{\prime}, \omega\right)
$$

and corresponding to the induced charges $\rho^{\text {ind }}$. In Eq. (A2), we use $\sigma\left(\mathbf{r}, \mathbf{r}^{\prime}, \omega\right)$ to denote the nonlocal conductivity tensor. In turn, this tensor corresponds to a nonlocal dielectric function

$$
\boldsymbol{\varepsilon}\left(\mathbf{r}, \mathbf{r}^{\prime}, \omega\right)=\mathbf{1}+\frac{4 \pi i}{\omega} \boldsymbol{\sigma}\left(\mathbf{r}, \mathbf{r}^{\prime}, \omega\right)
$$

Fourier transforming gives $\mathbf{E}_{\mathbf{k}}=-i \mathbf{q} \Phi_{\text {loc, }, \mathbf{q}}$, and a continuity specification

$$
\omega \rho_{\mathbf{q}}(\omega)=\mathbf{q} \cdot j_{\mathbf{q}}^{\text {ind }}
$$

that relates the local field and the induced charge

$$
4 \pi \rho_{\mathbf{q}}^{\text {ind }}(\omega)=-\mathbf{q} \cdot \sum_{\mathbf{q}^{\prime}}\left[\varepsilon_{\mathbf{q}, \mathbf{q}^{\prime}}(\omega)-\mathbf{1} \delta_{\mathbf{q}, \mathbf{q}^{\prime}}\right] \cdot \mathbf{q} \Phi_{\text {loc, } \mathbf{q}} \cdot
$$

We infer an exact, general microscopic relation between the (longitudinal) local-field response $\tilde{\chi}$ and the dielectric tensor

$$
\begin{aligned}
4 \pi \tilde{\chi}_{\mathbf{q}, \mathbf{q}^{\prime}} & =-\mathbf{q} \cdot\left[\boldsymbol{\varepsilon}_{\mathbf{q}, \mathbf{q}^{\prime}}-\mathbf{1} \delta_{\mathbf{q}, \mathbf{q}^{\prime}}\right] \cdot \boldsymbol{q}^{\prime}, \\
\tilde{\chi} & =\frac{1}{4 \pi} \nabla \cdot(\boldsymbol{\varepsilon}-\mathbf{1}) \cdot \nabla .
\end{aligned}
$$

The result Eq. (A6) reflects the continuity equation and identifies

$$
\boldsymbol{\varepsilon}_{\mathbf{q}, \mathbf{q}^{\prime}}^{\text {long }} \equiv \hat{\mathbf{q}} \cdot \boldsymbol{\varepsilon}_{\mathbf{q}, \mathbf{q}^{\prime}} \cdot \hat{\mathbf{q}}^{\prime},
$$

as the proper (consistent) definition of the longitudinal projection of the dielectric repose in an inhomogeneous system.

In Eq. (A7), the difference $(\boldsymbol{\varepsilon}-\mathbf{1}) / 4 \pi$ takes the form of a local-field susceptibility tensor $\sigma$. Using $\sigma_{\text {ext }}$ to denote the corresponding external-field susceptibility we also have a relation for the external-field response

$$
\chi=\nabla \cdot \sigma_{\mathrm{ext}} \cdot \nabla .
$$

Finally, the correct longitudinal projection of the dielectric tensor is given by

$$
\kappa \equiv \nabla \cdot \boldsymbol{\varepsilon} \cdot \nabla G=\varepsilon^{\text {long }} .
$$

This is demonstrated by expressing the microscopic relation in wave-vector space

$$
\begin{aligned}
4 \pi \tilde{\chi}_{\mathbf{q}, \mathbf{q}^{\prime}} & =-\mathbf{q} \cdot\left[\boldsymbol{\varepsilon}_{\mathbf{q}, \mathbf{q}^{\prime}}-\mathbf{1} \delta_{\mathbf{q}, \mathbf{q}^{\prime}}\right] \cdot \mathbf{q}^{\prime} \\
& =-|\mathbf{q}|\left|\mathbf{q}^{\prime}\right| \boldsymbol{\varepsilon}_{\mathbf{q}, \mathbf{q}^{\prime}}^{\text {long }}+q^{2} \delta_{\mathbf{q}, \mathbf{q}^{\prime}},
\end{aligned}
$$

where we have used the $\varepsilon^{\text {long }}$ specification Eq. (A8). Solving for $\boldsymbol{\varepsilon}_{\mathbf{q}, \mathbf{q}^{\prime}}^{\text {long }}$ gives

$$
\varepsilon_{\mathbf{q}, \mathbf{q}^{\prime}}^{\text {long }}=\delta_{\mathbf{q}, \mathbf{q}^{\prime}}-\frac{4 \pi}{|\mathbf{q}|\left|\mathbf{q}^{\prime}\right|} \tilde{\chi}_{\mathbf{q}, \mathbf{q}^{\prime}}
$$

[1] K. Rapcewicz and N. W. Ashcroft, Phys. Rev. B 44, 4032(R) (1991).

[2] O. Gunnarsson and B. I. Lundqvist, Phys. Rev. B 13, 4274 (1976).

[3] J. Harris and R. O. Jones, J. Phys. F.: Metal Phys. 4, 1170 (1974).
[4] O. Gunnarsson, M. Jonson, and B. I. Lundqvist, Phys. Rev. B 20, 3136 (1979).

[5] J. P. Perdew and D. C. Langreth, Sol. State. Commun. 17, 1425 (1975).

[6] D. C. Langreth and J. P. Perdew, Phys. Rev. B 15, 2884 (1977).

[7] Y. Wang and J. P. Perdew, Phys. Rev. B 44, 13298 (1991). 
[8] R. Eisenshitz and F. London, Z. Phys. 60, 491 (1930).

[9] F. London, Z. Phys. 63, 245 (1930).

[10] G. D. Mahan, J. Chem. Phys. 43, 1569 (1965).

[11] A. C. Maggs and N. W. Ashcroft, Phys. Rev. Lett. 59, 113 (1987).

[12] M. Rasolt and D. J. W. Geldart, Phys. Rev. Lett. 35, 1234 (1975); Phys. Rev. B 13, 1477 (1976).

[13] D. C. Langreth and J. P. Perdew, Phys. Rev. B 21, 5469 (1980).

[14] M. Rasolt and D. J. W. Geldart, Phys. Rev. B. 25, 5133 (1982).

[15] C. D. Hu and D. C. Langreth, Phys. Rev. B 33, 943 (1986).

[16] M. Rasolt and D. J. W. Geldart, Phys. Rev. B. 34, 1325 (1986).

[17] D. C. Langreth and S. H. Vosko, Phys. Rev. Lett. 59, 497 (1987).

[18] D. C. Langreth and S. H. Vosko, Adv. Quant. Chem. 21, 175 (1990).

[19] D. C. Langreth and M. J. Mehl, Phys. Rev. Lett. 47, 446 (1981).

[20] D. C. Langreth and J. P. Perdew, Phys. Rev. B 26, 2810 (1982).

[21] D. C. Langreth and M. J. Mehl, Phys. Rev. B 28, 1809 (1983).

[22] K. Burke, J. Chem. Phys. 136, 150901 (2012).

[23] A. D. Becke, J. Chem. Phys. 140, 18A301 (2014).

[24] Y. Andersson, D. C. Langreth, and B. I. Lundqvist, Phys. Rev. Lett. 76, 102 (1996).

[25] E. Hult, H. Rydberg, B. I. Lundqvist, and D. C. Langreth, Phys. Rev. B 59, 4708 (1999).

[26] E. Hult, Y. Andersson, B. I. Lundqvist, and D. C. Langreth, Phys. Rev. Lett. 77, 2029 (1996).

[27] H. Rydberg, B. I. Lundqvist, D. C. Langreth, and M. Dion, Phys. Rev. B 62, 6997 (2000).

[28] H. Rydberg, Ph.D. thesis, Chalmers University of Technology \& Göteborg University, Sweden, 2001.

[29] H. Rydberg, M. Dion, N. Jacobson, E. Schröder, P. Hyldgaard, S. I. Simak, D. C. Langreth, and B. I. Lundqvist, Phys. Rev. Lett. 91, 126402 (2003).

[30] M. Dion, Ph.D. thesis, Rutgers University, New Jersey, USA 2004.

[31] M. Dion, H. Rydberg, E. Schröder, D. C. Langreth, and B. I. Lundqvist, Phys. Rev. Lett. 92, 246401 (2004).

[32] M. Dion, H. Rydberg, E. Schröder, D. C. Langreth, and B. I. Lundqvist, Phys. Rev. Lett. 95, 109902(E) (2005)

[33] D. C. Langreth, M. Dion, H. Rydberg, E. Schröder, P. Hyldgaard, and B. I. Lundqvist, Int. J. Quantum Chem. 101, 599 (2005).

[34] T. Thonhauser, V. R. Cooper, S. Li, A. Puzder, P. Hyldgaard, and D. C. Langreth, Phys. Rev. B 76, 125112 (2007).

[35] V. R. Cooper, Phys. Rev. B 81, 161104(R) (2010).

[36] K. Lee, É. D. Murray, L. Kong, B. I. Lundqvist, and D. C. Langreth, Phys. Rev. B 82, 081101(R) (2010).

[37] K. Berland and P. Hyldgaard, Phys. Rev. B 87, 205421 (2013).

[38] K. Berland and P. Hyldgaard, Phys. Rev. B 89, 035412 (2014).

[39] K. Berland, C. A. Arter, V. R. Cooper, K. Lee, B. I. Lundqvist, E. Schröder, T. Thonhauser, and P. Hyldgaard, J. Chem. Phys. 140, 18A539 (2014).

[40] K. Berland, V. R. Cooper, K. Lee, E. Schröder, T. Thonhauser, P. Hyldgaard, and B. I. Lundqvist (unpublished).

[41] D. C. Langreth et al., J. Phys.: Condens. Matter 21, 084203 (2009).

[42] J. P. Perdew and Y. Wang, Phys. Rev. B 45, 13244 (1992).

[43] J. Schwinger, Phys. Rev. A 22, 1827 (1980); 24, 2353 (1981).

[44] P. Elliott and K. Burke, Can. J. Chem. 87, 1485 (2009).

[45] J. P. Perdew and Y. Wang, Phys. Rev. B 33, 8800 (1986).
[46] J. P. Perdew, K. Burke, and M. Ernzerhof, Phys. Rev. Lett. 77, 3865 (1996)

[47] Y. Zhang and W. Yang, Phys. Rev. Lett. 80, 890 (1998).

[48] É. D. Murray, K. Lee, and D. C. Langreth, J. Chem. Theory Comput. 5, 2754 (2009).

[49] J. Klimeš, D. R. Bowler, and A. Michaelides, Phys. Rev. B 83, 195131 (2011).

[50] I. Hamada, Phys. Rev. B 89, 121103 (2014).

[51] R. C. Clay, III, J. Mcminis, J.M. McMahon, C. Pierleoni, D.M. Ceperley, and M.A. Morales, Phys. Rev. B 89, 184106 (2014).

[52] M. A. Morales, J. R. Gergely, J. McMinis, J. M. McMahon, J. Kim, and D. M. Ceperley, J. Chem. Theory Comput. 10, 2355 (2014)

[53] K. Berland, E. Londero, E. Schröder, and P. Hyldgaard, Phys. Rev. B 88, 045431 (2013).

[54] A. Gulans, M. J. Puska, and R. M. Nieminen, Phys. Rev. B 79, 201105 (2009).

[55] G. Roman-Perez and J. M. Soler, Phys. Rev. Lett. 103, 096102 (2009).

[56] V. R. Cooper, T. Thonhauser, A. Puzder, E. Schröder, B. I. Lundqvist, and D. C. Langreth, J. Amer. Chem. Soc. 130, 1304 (2008).

[57] K. Berland and P. Hyldgaard, J. Chem. Phys. 132, 134705 (2010).

[58] E. Londero and E. Schröder, Phys. Rev. B 82, 054116 (2010).

[59] K. Berland, Ø. Borck, and P. Hyldgaard, Comp. Phys. Commun. 182, 1800 (2011).

[60] N. Nijem, P. Canepa, L. Kong, H. Wu, J. Li, T. Thonhauser, and Y. J. Chabal, J. Phys.: Condens. Matter 24, 424203 (2012).

[61] Y. Ihm, V. R. Cooper, L. Peng, and J. R. Morris, J. Phys.: Condens. Matter 24, 424205 (2012).

[62] P. Lazić, N. Atodiresei, R. Brako, B. Gumhalter, and S. Blügel, J. Phys.: Condens. Matt. 24, 424215 (2012).

[63] T. Björkman, A. Gulans, A. V. Krasheninnikov, and R. M. Nieminen, J. Phys.: Cond. Matter 24, 424218 (2012).

[64] J. Klimes and A. Michaelides, J. Chem. Phys. 137, 120901 (2012).

[65] R. Poloni, B. Smit, and J. B. Neaton, J. Phys. Chem. A 116, 4957 (2012).

[66] R. Poloni, K. Lee, R. F. Berger, B. Smit, and J. B. Neaton, J. Phys. Chem. Lett. 5, 861 (2014).

[67] T. Björkman, J. Chem. Phys. 141, 074708 (2014).

[68] All response functions and dielectric functions are nonlocal, i.e., functions of two spatial coordinates. The dependence is generally suppressed by considering equivalent operators $A\left(\omega ; \mathbf{r}, \mathbf{r}^{\prime}\right) \equiv\left\langle\mathbf{r}|A(\omega)| \mathbf{r}^{\prime}\right\rangle$. The Fourier transform is defined as $A\left(\omega ; \mathbf{q}, \mathbf{q}^{\prime}\right) \equiv \int d \mathbf{r} \int d \mathbf{r}^{\prime} e^{-i \mathbf{q} \cdot \mathbf{r}}\left\langle\mathbf{r}|A(\omega)| \mathbf{r}^{\prime}\right\rangle e^{i \mathbf{q}^{\prime} \cdot \mathbf{r}^{\prime}}$

[69] P. Nozières and D. Pines, Phys. Rev. 111, 442 (1958).

[70] B. I. Lundqvist, Phys. Kond. Mat. 6, 193 (1967).

[71] J. Lindhard, Mat. Fys. Medd. K. Dan. Vidensk. Selsk. 28, (1954).

[72] J. P. Perdew, L. A. Constantin, E. Sagvolden, and K. Burke, Phys. Rev. Lett. 97, 223002 (2006).

[73] L. A. Constantin, J. P. Perdew, and J. M. Pitarke, Phys. Rev. B 79, 075126 (2009).

[74] J. P. Perdew, K. Burke, and Y. Wang, Phys. Rev. B 54, 16533 (1996).

[75] V. Caciuc, N. Atodiresei, M. Callsen, P. Lazić, and S. Blügel, J. Phys.: Condens. Matt. 24, 424214 (2012).

[76] J. P. Perdew and Y. Wang, Phys. Rev. B 46, 12947 (1992). 
[77] J. P. Perdew, A. Ruzsinszky, G. I. Csonka, O. A. Vydrov, G. E. Scuseria, L. A. Constantin, X. Zhou, and K. Burke, Phys. Rev. Lett. 100, 136406 (2008).

[78] H. B. G. Casimir and D. Polder, Phys. Rev. 73, 360 (1948).

[79] K. Sawada, Phys. Rev. 106, 372 (1957); K. Sawada, K. A. Brueckner, N. Fukuda, and R. Brout, ibid. 108, 507 (1957).

[80] For an introduction to RPA calculations of the HEG groundstate energy, see David Pines, Elementary excitations in Solids (Addison-Wesley Publishing Company, Inc, Redwood City, California, 1963), Appendix C.

[81] F. Furche, J. Chem. Phys. 129, 114105 (2008).

[82] H. Eshuis, J. E. Bates, and F. Furche, Theor. Chem. Acc. 131, 1084 (2012).

[83] X. Ren, P. Rinke, C. Joas, and M. Scheffler, J. Mater. Sci. 47, 7447 (2012).

[84] J. Harris, Phys. Rev. B 31, 1770 (1985).

[85] K. H. Lau and W. Kohn, Surf. Sci. 75, 69 (1978).

[86] T. L. Einstein, Surf. Sci. 75, 161L (1978).

[87] P. Hyldgaard and M. Persson, J. Phys.:Condens. Matt. 12, L13 (2000).

[88] J. Repp, F. Moresco, G. Meyer, K.-H. Rieder, P. Hyldgaard, and M. Persson, Phys. Rev. Lett. 85, 2981 (2000).

[89] P. Hyldgaard and T. L. Einstein, Eur. Phys. Lett. 59, 265 (2002).

[90] K. Berland, T. L. Einstein, and P. Hyldgaard, Phys. Rev. B 80, 155431 (2009).

[91] P. Hyldgaard, J. Phys.: Condens. Matter 24, 424219 (2012).

[92] B. M. Axilrod and E. Teller, J. Chem. Phys. 11, 299 (1943).

[93] Y. U. Barash, Fiz. Tverd. Tela (Leningrad) 30, 1578 (1988) [Sov. Phys. Solid State 30, 1580 (1988)].

[94] Y. U. Barash and O. I. Notysh, Zh. Exp. Tero. Fiz. 98, 502 (1990) [Sov. Phys. JETP 71, 301 (1990)].

[95] B. E. Sernelius and P. Björk, Phys. Rev. B 57, 6592 (1998).

[96] M. Boström and B. E. Sernelius, Phys. Rev. B 61, 2204 (2000).

[97] J. F. Dobson, A. White, and A. Rubio, Phys. Rev. Lett. 96, 073201 (2006).

[98] A. Tkatchenko and O.A. von Lilienfeld, Phys. Rev. B 78, 045116 (2008).
[99] S. Lebégue, J. Harl, T. Gould, J. G. Ángyán, G. Kresse, and J.F. Dobson, Phys. Rev. Lett. 105, 196401 (2010).

[100] A. Tkatchenko, R. A. DiStasio, Jr., R. Car, and M. Scheffler, Phys. Rev. Lett. 108, 236402 (2012).

[101] A. Ruzsinszky, J. P. Perdew, J. Tao, G. I. Csonka, and J. M. Pitarke, Phys. Rev. Lett. 109, 233203 (2012).

[102] J. P. Perdew, J. Tao, P. Hao, A. Ruzsinszky, G. I. Csonka, and J. M. Pitarke, J. Phys.: Condens. Matter 24, 424207 (2012).

[103] J. F. Dobson and T. Gould, J. Phys.: Condens. Matter 24, 073201 (2012).

[104] V. V. Gobre and A. Tkatchenko, Nat. Commun. 4, 2341 (2013).

[105] J. F. Dobson, T. Gould, and G. Vignale, Phys. Rev. X 4, 021040 (2014).

[106] J. F. Dobson, Int. J. Quantum Chem. 114, 1157 (2014).

[107] J. Kleis, E. Schröder, and P. Hyldgaard, Phys. Rev. B 77, 205422 (2008).

[108] K. Lee, A. K. Kelkkanen, K. Berland, S. Andersson, D. C. Langreth, E. Schröder, B. I. Lundqvist, and P. Hyldgaard, Phys. Rev. B 84, 193408 (2011).

[109] K. Lee, K. Berland, M. Yoon, S. Andersson, E. Schröder, P. Hyldgaard, and B. I. Lundqvist, J. Phys.: Condens. Matter 24, 424213 (2012).

[110] The hollow $\mathrm{C}_{60}$, formed by a shell with a near-metallic nature [101, 102], supplements graphene and metallic nanotubes as examples where the modern expanded vdW-DF versions cannot capture the vdW interaction across all length scales. In $\mathrm{C}_{60}$ screening leads to the creation of a $l=0$ plasmon excitation of vanishing frequency and a per-fragment response that differs markedly from that obtained by treating the molecular fragments at the GGA level [101, 102]. When studying the $\mathrm{vdW}$ attraction at larger separations it is not possible to both truncate the screening and represent these hollow/extended structures as just a single molecular-type fragment with a GGA-type screening (as the recent vdW-DF versions do) [99,101-103,105].

[111] S. D. Chakarova-Käck, A. Vojvodic, J. Kleis, P. Hyldgaard, and E. Schröder, New J. Phys. 12, 013017 (2010). 